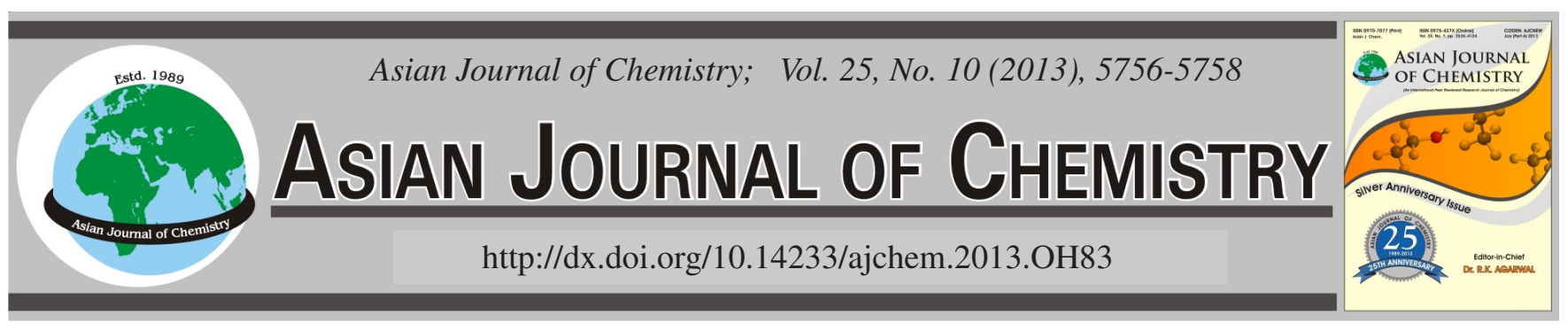

\title{
Adsorption of Methylene Blue on Carbon Nanotubes from Aqueous Solutions $\dagger$
}

\author{
Donglin Zhao*, Yi Ding ${ }^{*}$, Shaohua Chen, Tingting Bai and Yunlei Ma
}

School of Materials and Chemical Engineering, Anhui University of Architecture, Hefei 230601, P.R. China

*Corresponding author: E-mail: zhaodlin@126.com; dyrqf@aiai.edu.cn

\begin{abstract}
In this study, the adsorption of methylene blue on carbon nanotubes from aqueous solutions has been investigated in a batch process. Experiments have been carried out to investigate the effects of temperature, methylene blue concentration, $\mathrm{pH}$, carbon nanotubes mass on methylene blue adsorption. The experimental results show that the methylene blue adsorption rate increases with its concentration in the aqueous solutions. Methylene blue adsorption also increases with temperature. The removal of methylene blue from the solution increases as carbon nanotubes mass increases. The kinetic study manifests that the adsorption of methylene blue on the carbon nanotubes is in good compliance with the pseudo-second-order kinetic model.
\end{abstract}

Key Words: Adsorption kinetics, Carbon nanotube, Methylene blue, Thermodynamics.

\section{INTRODUCTION}

Dyes are widely used in industries such as the textile, pulp mill, paper, dye synthesis, leather, food, printing and plastics industries. Since many of the organic dyestuffs are toxic to microorganisms and harmful to human beings, removal of dyestuffs from wastewater has received considerable attention over the past decades ${ }^{1,2}$. Most of these dyes cause serious problems for the ecological system, as they are toxic and have carcinogenic properties, which make the water contaminated with dyes inhibitory to aquatic living organisms ${ }^{3}$.

Methylene blue dye gives rise to eye burns, which may be responsible for permanent hurt to the eyes of human and animals ${ }^{4}$. On inhalation, it can cause short periods of difficult or rapid breathing, but ingestion through the mouth produces a burning sensation and may cause nausea, profuse sweating, mental confusion, vomiting, painful micturition and methemoglobinemia ${ }^{5,6}$. Therefore the treatment of effluent containing such dye is of great interest owing to its effects on receiving waters.

Carbon nanotubes have attracted great interest in multidisciplinary areas owing to their unique hollow tube structure and various potential applications ${ }^{7,8}$. Carbon nanotubes have been proposed for different applications such as sensors, hydrogen storage devices and so on ${ }^{9,10}$. Because of their easily modified surfaces and large surface areas, extensive experiments have been conducted on the adsorption of organic or inorganic contaminants on carbon nanotubes ${ }^{11,12}$. Methylene blue is the cationic dye that is most commonly used for colouring. This dye has been investigated due to its known strong adsorption onto solids and it usually serves as a model compound for removing organic pollutants and colored bodies from aqueous solutions. Therefore, methylene blue was employed as the organic contaminants to be treated by carbon nanotubes in this work.

Understanding the kinetic of the adsorption is critical for the development of more efficient adsorbents suitable for real environmental applications. In this work, the adsorption kinetics of methylene blue on MWCNTs were studied. The objective of this study is to investigate the effects of methylene blue concentration, temperature, $\mathrm{pH}$, carbon nanotubes mass on the adsorption process. The kinetic study shows that methylene blue adsorption on the MWCNTs is in good compliance with the pseudo-second-order kinetic model.

\section{EXPERIMENTAL}

MWCNTs was prepared according to a method described in a previous study ${ }^{13}$. All other chemicals were purchased in analytical purity and used without further purification and all solutions were prepared with Milli-Q water under ambient conditions.

Analysis methods: The adsorbate concentrations in the initial and final aqueous solutions were measured by using UV-VIS spectrophotometer at $665 \mathrm{~nm}$. The amount of methylene blue adsorbed on MWCNTs was calculated from the difference

$†$ Presented to the 6th China-Korea International Conference on Multi-functional Materials and Application, 22-24 November 2012, Daejeon, Korea 
between the initial concentration and the equilibrium one. The adsorption percentage (adsorption $\left.\%=\left(\mathrm{C}_{0}-\mathrm{C}_{\mathrm{e}}\right) / \mathrm{C}_{0} \times 100 \%\right)$ was derived from the difference of the initial concentration $\left(\mathrm{C}_{0}\right)$ and the final one $\left(\mathrm{C}_{\mathrm{e}}\right)$ in supernatant after centrifugation. All experimental data were the average of triplicate determinations and the relative errors were about $\pm 5 \%$.

\section{RESULTS AND DISCUSSION}

Effect of MWCNTs mass on methylene blue adsorption: To study the effect of MWCNTs mass on methylene blue adsorption, $10 \mathrm{mg} / \mathrm{L}$ methylene blue solutions were mixed with different MWCNTs mass for $130 \mathrm{~min}$, while keeping other parameters constant. It can be seen from the experimental results depicted in Fig. 1 that methylene blue adsorption on per gram of MWCNTs decreases as the MWCNTs mass in the solution increases. For example, after 130 min operation, the methylene blue adsorption decreases from 73.5 to 43.7 $\mathrm{mg} / \mathrm{g}$ as MWCNTs mass increases from $0.1 \mathrm{~g} / \mathrm{L}$ to $0.2 \mathrm{~g} / \mathrm{L}$. Such performance on the adsorbent may be attributed to the fact that some of the adsorption sites remain unsaturated during the adsorption process. As the initial methylene blue concentration keeps constant, it is noted that the total adsorptive capability of methylene blue increases faster as the MWCNTs mass increases due to the increase in the number of reaction sites and surface area available to methylene blue.

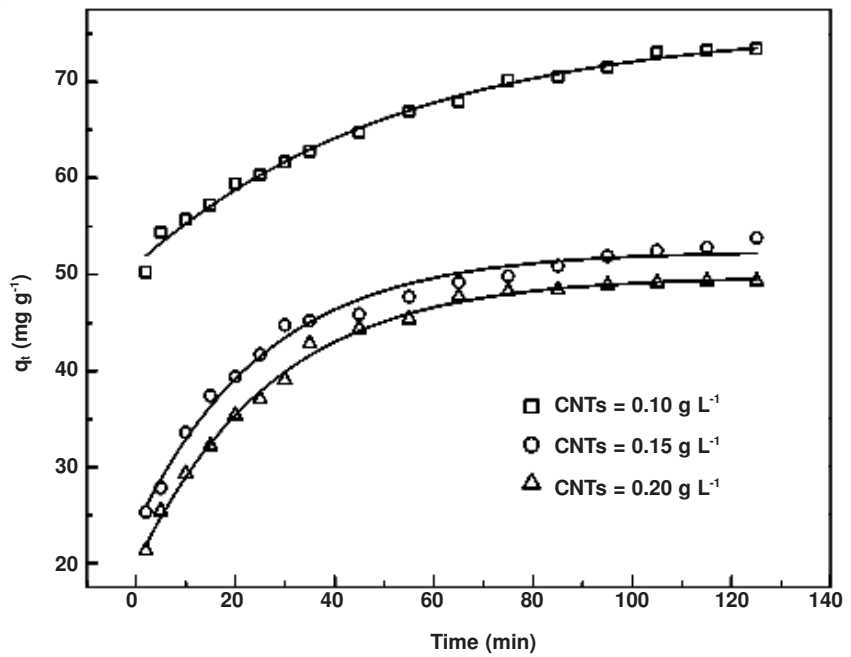

Fig. 1. Effect of MWCNTs mass on adsorption ofmethylene blue adsorption

Effect of methylene blue concentration on its adsorption: Experiments have been performed to test the effect of methylene blue concentration on its adsorption on MWCNTs. The experimental results shown in Fig. 2 manifest that the methylene blue adsorption on MWCNTs increases with its concentration in the aqueous solutions. The explanation can be expressed as follows. Firstly, the mass-transfer impetus between the liquid bulk and the surface of MWCNTs increases with the initial methylene blue concentration in the aqueous solution. Secondly, the diffusivity of methylene blue also increases with its concentration. Thirdly, the methylene blue on the surface of MWCNTs in equilibrium with the methylene blue in the solution will increase with its concentration in the solution $^{14}$.

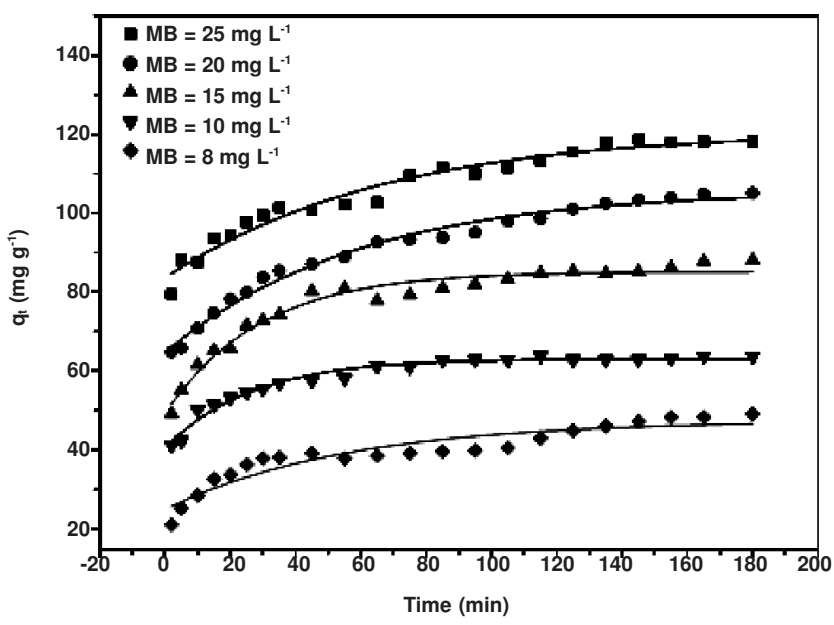

Fig. 2. Effect of concentration on adsorption of methylene blue

Effect of pH on adsorption of methylene blue: Effect of $\mathrm{pH}$ on the adsorption of methylene blue on MWCNTs is shown in Fig. 3. Fig. 3 shows that the $\mathrm{pH}$ value on methylene blue adsorption cannot be ignored. As $\mathrm{pH}$ increases, the equilibrium adsorbate increases. As the initial $\mathrm{pH}$ value increases from 6.5 to 9.4 , the equilibrium adsorbate increases from 53.3 $\mathrm{mg} / \mathrm{g}$ to $64.1 \mathrm{mg} / \mathrm{g}$. The explanation can be expressed as follows. MWCNTs surface has a weak polarity under different $\mathrm{pH}$ value. In the acidic conditions, the surface of MWCNTs is positively charged, which is unfavorable for the adsorption of cation dye. As $\mathrm{pH}$ rises, the surface of MWCNTs gradually becomes negative charged, which may result in the increase of methylene blue adsorption.

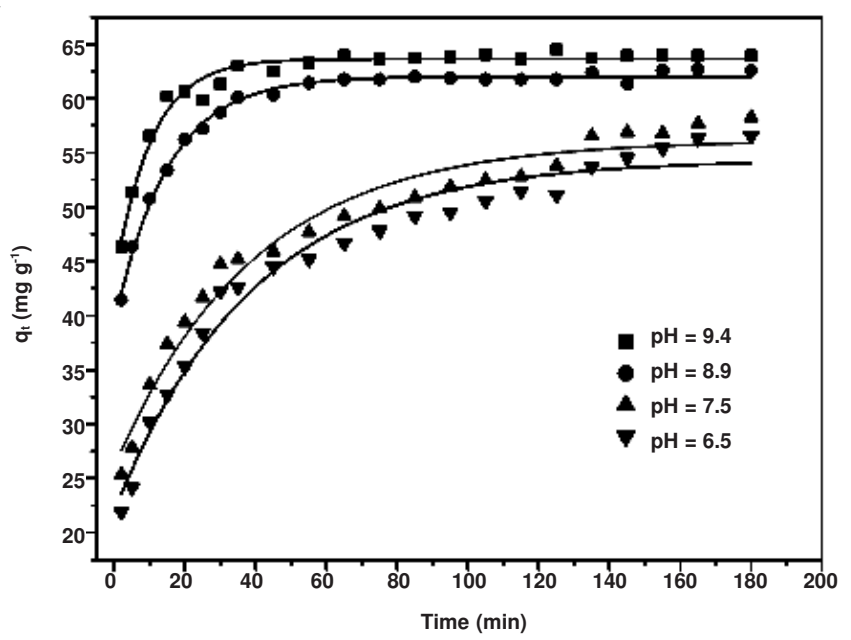

Fig. 3. Effect of $\mathrm{pH}$ on adsorption of methylene blue

\section{Adsorption dynamics}

Pseudo-first-order kinetic model: The pseudo-first-order kinetic model has been widely used to predict adsorption kinetics. The pseudo-first-order kinetic mode ${ }^{15}$ describes the adsorption of liquid/solid system based on solid capacity. The model can be written as:

$$
\log \left(\mathrm{q}_{\mathrm{e}}-\mathrm{q}_{\mathrm{t}}\right)=\log \mathrm{q}_{\mathrm{e}}-\frac{\mathrm{k}_{1}}{2.303} \mathrm{t}
$$

where $\mathrm{q}_{\mathrm{e}}$ and $\mathrm{q}_{\mathrm{t}}$ is the capacity of metal ions adsorbed $\left(\mathrm{mg} \mathrm{g}^{-1}\right)$ at equilibrium and time $\mathrm{t}(\mathrm{h})$, respectively and $\mathrm{K}_{1}$ is the pseudo- 
first-order rate constant $\left(\mathrm{h}^{-1}\right)$. Thus the values of $\mathrm{q}_{\mathrm{e}}$ and $\mathrm{k}_{1}$ can be determined experimentally by plotting $\log \left(\mathrm{q}_{\mathrm{e}}-\mathrm{q}_{\mathrm{t}}\right)$ versus $\mathrm{t}$ and extracting information from the least squares analysis of slope and intercept and substituting into eqn. (1). The plots of $\log \left(\mathrm{q}_{\mathrm{e}}-\mathrm{q}_{\mathrm{t}}\right)$ versus $\mathrm{t}$ are shown in Fig. 4 . The calculated $\mathrm{q}_{\mathrm{e}}, \mathrm{k}_{1}$, and the corresponding linear regression correlation coefficient $\mathrm{R}^{2}$ values are shown in Table- 1 .

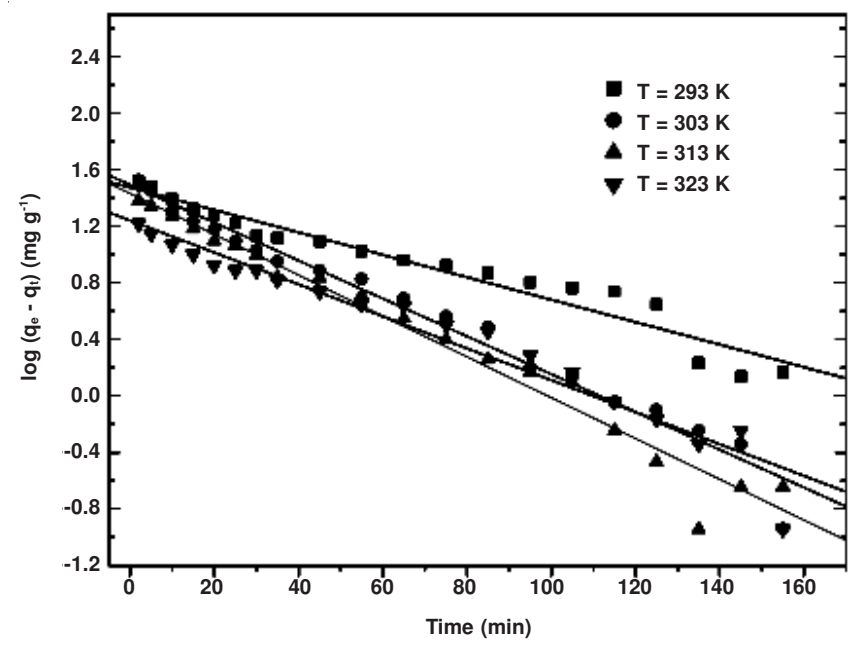

Fig. 4. Pseudo-first-order kinetics for adsorption of methylene blue onto MWCNTs

TABLE-1

KINETIC PARAMETERS FOR THE ADSORPTION OF MB ON MWCNTS AT VARIOUS TEMPERATURES

\begin{tabular}{lllll}
\hline & $293 \mathrm{~K}$ & $303 \mathrm{~K}$ & $313 \mathrm{~K}$ & $323 \mathrm{~K}$ \\
\hline Pseudo-first-prder & & & & \\
\hline $\mathrm{K}_{1}\left(\mathrm{~min}^{-1}\right)$ & 0.018 & 0.031 & 0.033 & 0.035 \\
$\mathrm{q}_{\mathrm{e}}\left(\mathrm{mg} \cdot \mathrm{g}^{-1}\right)$ & 47.07 & 56.23 & 57.44 & 61.32 \\
$\mathrm{R}^{2}$ & 0.9414 & 0.9758 & 0.9717 & 0.9494 \\
\hline Pseudo-second-order & & & & \\
\hline $\mathrm{K}_{2}\left(\mathrm{~g} \cdot \mathrm{mg}^{-1} \cdot \mathrm{min}^{-1}\right)$ & $1.58 \mathrm{E}-3$ & $2.48 \mathrm{E}-3$ & $3.06 \mathrm{E}-3$ & $4.27 \mathrm{E}-3$ \\
$\mathrm{qe}\left(\mathrm{mg} \cdot \mathrm{g}^{-1}\right)$ & 59.88 & 63.29 & 64.52 & 65.36 \\
$\mathrm{R}^{2}$ & 0.9955 & 0.9994 & 0.9995 & 0.9995 \\
\hline
\end{tabular}

Pseudo-second-order kinetic model: The pseudo-secondorder adsorption kinetic model is expressed as following formulation ${ }^{16}$ :

$$
\frac{\mathrm{t}}{\mathrm{q}_{\mathrm{t}}}=\frac{1}{\mathrm{k}_{2} \mathrm{q}_{\mathrm{e}}{ }^{2}}+\frac{1}{\mathrm{q}_{\mathrm{e}}} \mathrm{t}
$$

Thus the values of $\mathrm{k}_{2}$ and $\mathrm{q}_{\mathrm{e}}$ can be calculated from the intercept and the slope of the linear relationship, eqn. (2), between $t / q_{t}$ and $t$. The curves of the plots of $t / q_{t}$ versus $t$ were given in Fig. 5 and the calculated $\mathrm{q}_{\mathrm{e}}, \mathrm{k}_{2}$ and the corresponding linear regression correlation coefficient $\mathrm{R}^{2}$ values are summarized in Table-1. The correlation coefficients for the secondorder kinetics model $\left(\mathrm{R}^{2}\right)$ are greater than 0.99 , indicating the applicability of this kinetics equation and the second-order nature of the adsorption process of methylene blue onto MWCNTs. From the relative coefficient, it can be seen that the pseudo-second-order kinetic model fit the adsorption of methylene blue onto MWCNTs better than the pseudo-firstorder model.

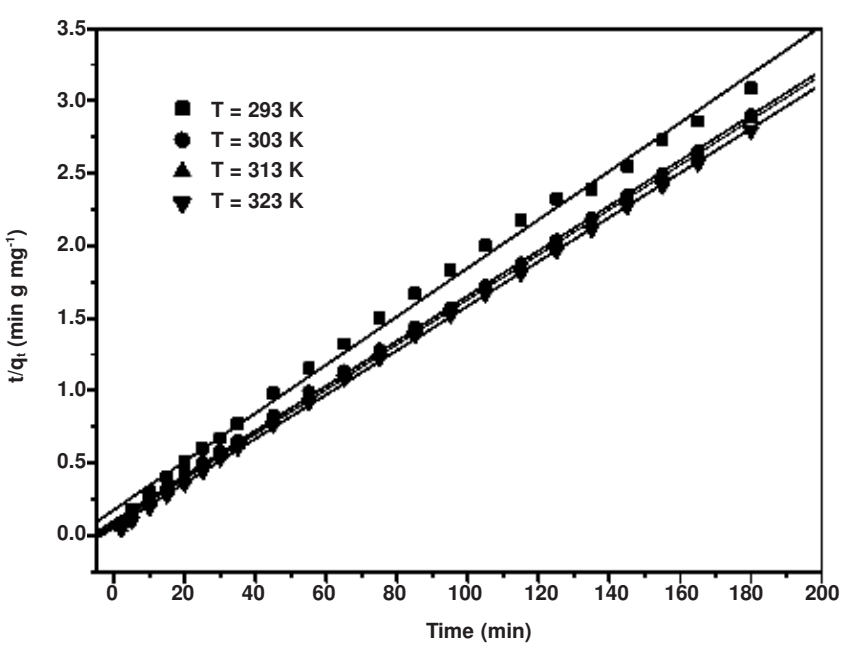

Fig. 5. Pseudo-second-order kinetics for adsorption of methylene blue onto MWCNTs

\section{Conclusion}

The adsorption of methylene blue onto MWCNTs is favored at high $\mathrm{pH}$ and higher temperature. The removal of methylene blue by adsorption on MWCNTs was found to be rapid at the initial period of contact time and then slows down with increasing reaction time. Adsorption data were modeled using the first and second-order kinetic equations. The secondorder kinetic equation could best describe the adsorption kinetics.

\section{ACKNOWLEDGEMENTS}

Financial support from the PhD Start-up Fund of Anhui University of Architecture and the National Innovation Foundation for College Students (201210878050) are acknowledged.

\section{REFERENCES}

1. C.H. Weng and Y.F. Pan, J. Hazard. Mater., 144, 355 (2007).

2. M.M. Alexandro, A.L. Cazetta, M.H. Kunita, T.L. Silva and V.C. Almeida, Chem. Eng. J., 168, 722 (2011).

3. K.R. Ramakrishna and T. Viraraghavan, Water Sci. Technol., 36, 189 (1997).

4. B.H. Hameed, A.T.M. Din and A.L. Ahmad, J. Hazard. Mater., 141, 819 (2007).

5. D. Ghosh and K.G. Bhattacharyya, Appl. Clay Sci., 20, 295 (2002).

6. J. Avom, J.K. Mbadcam, C. Noubactep and P. Germain, Carbon, 35, 365 (1997).

7. N. Jonge, Y. Lamy, K. Schoots and T.H. Oosterkamp, Nature, 420, 393 (2002).

8. M.S. Dresselhaus and I.L. Thomas, Nature, 414, 332 (2001).

9. M.S. Mauter and M. Elimelech, Environ. Sci. Technol., 42, 5843 (2008).

10. J. Wang, C. Timchalk and Y. Lin, Environ. Sci. Technol., 42, 2688 (2008).

11. G.P. Rao, C. Lu and F. Su, Sep. Purif. Technol., 58, 224 (2007).

12. B. Pan and B.S. Xing, Environ. Sci. Technol., 42, 9005 (2008).

13. G.D. Sheng, D.D. Shao, X.M. Ren, X.Q. Wang, J.X. Li, Y.X. Chen and X.K. Wang, J. Hazard. Mater., 178, 505 (2010).

14. H.S. Zhu, X.J. Yang, Y.P. Mao, Y. Chen, X.L. Long and Wei-kang Yuan, J. Hazard. Mater., 185, 951 (2011).

15. S. Lagergren, Kung, Sven. Veten. Hand., 24, 1 (1898).

16. Y.S. Ho, Ph. D. Thesis, Adsorption of Heavy Metals from Waste Streams by Peat, The University of Birmingham, Birmingham, UK (1995). 